Jan J. De Waele

Matteo Bassetti

Ignacio Martin-Loeches

\section{Impact of de-escalation on ICU patients' prognosis}

Received: 22 August 2014

Accepted: 4 September 2014

Published online: 13 September 2014

(C) Springer-Verlag Berlin Heidelberg and ESICM 2014

J. J. De Waele (凶)

Department of Critical Care Medicine, Ghent University Hospital, De Pintelaan 185, 9000 Ghent, Belgium

e-mail: jan.dewaele@ugent.be

\section{Bassetti}

Azienda Ospedaliera Universitaria Santa Maria della Misericordia, Udine, Italy

\section{Martin-Loeches}

St James's University Hospital, Trinity Centre for Health Sciences, Multidisciplinary Intensive Care Research Organization (MICRO), St James Street, Dublin 7, Ireland

De-escalation has been advocated to reduce antibiotic pressure and has become an integrated part of antimicrobial stewardship programs and antimicrobial therapy guidelines [1]. The goal of de-escalation is to reduce the use of broad-spectrum antibiotics when bacterial susceptibility allows this. As such, it has become a necessity as proposed empirical antibiotic therapy for severe infection most often consists of multiple and broad-spectrum antibiotics. The application of de-escalation in real life is rather disappointing and ranges from 13 to $43 \%$ [2-6] depending on the definition used and context studied.

Although reducing antibiotic use is an essential strategy to reduce antimicrobial pressure, it is important that it remains safe from the patient's perspective. It can be expected that avoiding potential side effects by withholding antibiotics not required for appropriate therapy may have a beneficial effect on outcome. In the past, most studies on de-escalation could not find a deleterious effect on outcome, and some studies even suggested that deescalation is beneficial [7] although the reasons why remain elusive. All studies in this respect were noninterventional, most were retrospective, and as such, a selection bias was inevitable. It can be assumed that deescalation was only considered if patients improved and thus de-escalation itself was a marker of clinical improvement. This is also suggested by the fact that escalation of antibiotic therapy was associated with worse outcome in these studies [3,7].

The current study by Leone et al. [8] casts significant doubt whether the reduction of the spectrum of the antibiotic can be considered safe as a routine measure. In their randomized controlled trial (RCT), the authors demonstrated that de-escalation, defined as narrowing the spectrum of the antibiotic, was inferior to continuation of the initial antibiotic therapy with length of stay as the primary outcome parameter. Furthermore, antibiotic use was higher in the de-escalation group presumably driven by the number of superinfections in the de-escalation group.

Obviously there are a number of limitations in the study by Leone et al. that make drawing firm conclusions difficult. This was an unblinded trial as keeping the antibiotic treatment concealed for the treating physician would be difficult, and associated bias cannot be completely excluded. There were indeed the imbalances between the two groups in severity of illness. However this was an advantage - if any - primarily for the de-escalation group as patients were younger and had lower severity of illness at the start, and one would expect the length of stay (the primary endpoint) to be shorter in younger and less severely ill patients. There was also a trend towards more pulmonary infections in the de-escalation group but a separate analysis of patients with pulmonary infections only could not find any differences. Finally, de-escalation patients were treated with carbapenems as an empirical strategy more often, but it is unclear how this could have 
affected outcome. One of the strengths of the study was that they included all patients in whom de-escalation could be considered and therefore did not suffer from the selection bias previously discussed.

The results of this study are in contrast with two papers recently published in Intensive Care Medicine [7, 8]. In both papers the outcome in terms of mortality rates in deescalated patients was at least not worse than those not de-escalated. In a study investigating febrile neutropenia patients, observation was extended to 1 year after intensive care unit discharge, which was the longest recorded follow-up period in the literature [9]. This did not alter the results. Another study demonstrated a protective effect of de-escalation in terms of mortality (odds ratio, 0.54; $95 \%$ confidence interval, 0.33-0.89) [7].

On the basis of the disparate findings by Leone et al. it could therefore be questioned if de-escalation-defined as changing the drug purely to narrow the spectrum - is a safe and sensible approach. The presumed safety suggested in other studies could not be confirmed and in particular the increase in antibiotic use is worrying. In this respect, deescalation failed its main goals - reducing antibiotic use and improving (or at least safeguarding) patient outcome.

The reasons for this are at this point unclear. The high frequency of colonization with less susceptible organisms commonly encountered in critically ill patients could be an explanation-when the spectrum is reduced these organisms may get an ecological advantage. But almost half of the superinfections in the de-escalation group were caused by the same pathogen as the primary infection. Apparently, the drug used in de-escalation was not adequate in eradicating the organism. This could be due to differences in susceptibility or limited knowledge about the PK/PD of these narrow spectrum antibiotics in critically ill patients.

Despite the current findings, de-escalation requires further study and the definition of de-escalation in this context is pivotal. In all studies different strategies have been considered de-escalation, such as (a) reduction of the number of antibiotics, (b) reduction of the spectrum of the antibiotic, (c) stopping combination therapy, and even
Table 1 Preferred strategies to reduce antibiotic consumption in critically ill patients

1.Use of adequate and rapid diagnostic tools to avoid antibiotic use altogether when there is no infection present

2 . Reduction of treatment duration for established infections

5-7 days for most indications

3. Discontinuation of antibiotics that do not cover the causative pathogen, as this is unnecessary and dangerous. Also continuing combination therapy beyond the first days should be avoided

(d) limiting the duration of therapy. The impact of these different approaches may be different and it is questionable if they should all be considered de-escalation. Deescalation as narrowing the spectrum of the antibioticthe strictest definition as was studied by Leone et al. [8]probably has the lowest impact on antibiotic exposure. Inevitably, the patients will have been treated with empirical therapy for several days (median 3 days in the current study, comparable to previous studies [7]) before de-escalation is applied. This may be enough to induce resistance. Furthermore, the patient is exposed to two different antibiotics - often from different classes-during one treatment, which may enhance rather than prevent resistance.

The goals of de-escalation, however, remain relevant and reducing antibiotic use is a key element to stop (or at least slow down) antibiotic resistance [10]. The question, however, is if other strategies are not better suited for this. The preferred antibiotic stewardship interventions to reduce antibiotic use in critically ill patients are listed in Table 1

In conclusion, the safety of de-escalation in terms of preserving outcome and reducing antibiotic use has been challenged in a recent RCT. A key element in the study of the potential role of de-escalation is a uniform definition of de-escalation. De-escalation-defined as narrowing the spectrum of an antibiotic treatment-should be cautiously applied, based on each particular patient's clinical status and considering the ICU environment as a whole.

\section{References}

1. Dellinger RP, Levy MM, Rhodes A, Annane D, Gerlach H, Opal SM, Sevransky JE, Sprung CL, Douglas IS, Jaeschke R, Osborn TM, Nunnally ME, Townsend SR, Reinhart K, Kleinpell RM, Angus DC, Deutschman CS, Machado FR, Rubenfeld GD, Webb S, Beale RJ, Vincent JL, Moreno R (2013) Surviving sepsis campaign: international guidelines for management of severe sepsis and septic shock, 2012. Intensive Care Med $39: 165-228$
2. Rello J, Vidaur L, Sandiumenge A, Rodriguez A, Gualis B, Boque C, Diaz E (2004) De-escalation therapy in ventilator-associated pneumonia. Crit Care Med 32:2183-2190

3. Kollef MH, Morrow LE, Niederman MS, Leeper KV, Anzueto A, BenzScott L, Rodino FJ (2006) Clinical characteristics and treatment patterns among patients with ventilatorassociated pneumonia. Chest 129:1210-1218
4. De Waele JJ, Ravyts M, Depuydt P, Blot SI, Decruyenaere J, Vogelaers D (2010) De-escalation after empirical meropenem treatment in the intensive care unit: fiction or reality? J Crit Care 25:641-646

5. Heenen S, Jacobs F, Vincent JL (2012) Antibiotic strategies in severe nosocomial sepsis: why do we not deescalate more often? Crit Care Med 40:1404-1409 
6. Montravers P, Dupont H, Gauzit R, Veber B, Bedos JP, Lepape A (2011) Strategies of initiation and streamlining of antibiotic therapy in 41 French intensive care units. Crit Care 15:R17

7. Garnacho-Montero J, GutierrezPizarraya A, Escoresca-Ortega A, Corcia-Palomo Y, Fernandez-Delgado E, Herrera-Melero I, Ortiz-Leyba C, Marquez-Vacaro JA (2014) Deescalation of empirical therapy is associated with lower mortality in patients with severe sepsis and septic shock. Intensive Care Med 40:32-40
8. Leone M, Bechis C, Baumstarck K, Lefrant JY, Albanese J, Jaber S, Lepape A, Constantin JM, Papazian L, Bruder $\mathrm{N}$, Allaouchiche B, Bezulier K, Antonini F, Textoris J, Martin C (2014) De-escalation versus continuation of empirical antimicrobial treatment in severe sepsis: a multicenter non-blinded randomized noninferiority trial. Intensive Care Med. doi: 10.1007/s00134-014-3411-8
9. Mokart D, Slehofer G, Lambert J, Sannini A, Chow-Chine L, Brun JP, Berger P, Duran S, Faucher M, Blache JL, Saillard C, Vey N, Leone M (2014) De-escalation of antimicrobial treatment in neutropenic patients with severe sepsis: results from an observational study. Intensive Care Med 40:41-49

10. Martin-Loeches I, Diaz E, Valles J (2014) Risks for multidrug-resistant pathogens in the ICU. Curr Opin Crit Care 20:516-524 